\title{
La production de carburants à partir de biomasse lignocellulosique par voie biologique : état de l'art et perspectives
}

\author{
Michael J. O'DONOHUE \\ Laboratoire d'ingénierie des systèmes \\ biologiques et des procédés, \\ INSA UMR INRA 792/CNRS 5504, \\ 135, avenue de Rangueil, \\ 31077 Toulouse cedex 04 \\ $<$ michael.odonohue@insa-toulouse.fr>
}

\begin{abstract}
In the current international context, the development of industrial technology that will allow the production of agro-fuels from lignocellulosic biomass is becoming ever more important for society. However, despite over thirty years of research, several hurdles still have to be surmounted before the first industrial facilities begin to produce ethanol. Nevertheless, thanks to its ability to generate innovative, clean and sustainable solutions for industry, biotechnology is now well-poised to provide new solutions aimed at the full exploitation of biomass resources, not only to make fuels, but also a wide range of fine chemicals and products that are required by modern society.
\end{abstract}

Key words: biotechnology, fuels, lignocellulosic biomass, research
Une contribution aux Comptes rendus de I'Académie des Sciences de 1910 écrite par MM Ville et Mestrezat [1] nous rappelle que la recherche sur la transformation de la cellulose en éthanol était déjà, il y a un siècle, une vieille histoire. Cependant, c'est surtout depuis la première crise pétrolière dans les années 70 que les recherches visant la production d'éthanol à partir de la cellulose se sont véritablement intensifiées. Depuis cette époque, trois verrous liés au prétraitement de la biomasse, à l'hydrolyse enzymatique de la cellulose et à la fermentation des sirops complexes ont focalisé la plupart des efforts. Dans cette revue, nous ferons état du progrès réalisé dans ces domaines et nous évoquerons le rôle important que la biotechnologie jouera dans les évolutions futures.

\section{Les limites \\ de l'éthanol-carburant de première génération}

Aujourd'hui, l'ère de l'éthanol-carburant est arrivée. En l'espace de quelques années, l'image (souvent fausse) du Brésil avec son industrie de l'éthanol-carburant un peu désuète a été balayée et remplacée par celle des États-Unis avec sa nouvelle production d'éthanol à partir du maïs [2]. Cependant, peu de choses permettent de différencier ces industries. Notamment, elles fonctionnent toutes les deux avec des technologies bien établies qui utilisent des glucides végétaux de réserve (le saccharose et l'amidon respectivement) comme matière première. Pour le saccharose, il s'agit du produit de la canne à sucre ou de la betterave à sucre, alors que pour l'amidon il s'agit essentiellement du maïs ou du blé. En Europe, où l'éthanol est souvent puisé des deux sources simultanément, l'industrie de l'éthanol-carburant s'est développée rapidement et constitue désormais une pierre angulaire de la politique énergétique de la Commission européenne ainsi que de plusieurs des États membres. Selon les estimations pour I'année 2007, la France aurait produit entre 550 et 600 millions de litres d'éthanolcarburant, ce qui lui permettrait d'atteindre son objectif fixé à 3,5\% du bouquet énergétique [3].

Néanmoins, malgré les avantages de ce carburant fabriqué à partir de ressources renouvelables, ses détracteurs n'ont pas de difficultés pour identifier les points négatifs. D'abord, la manufacture de ce carburant constitue une menace pour le bon fonctionnement de la filière agroalimentaire, car - provenant de l'utilisation des glucides de réserve - il constitue une utilisation alternative pour des denrées alimentaires telles que le blé, le maïs (grains) et le saccharose. De même, de nombreuses études portant sur le bilan $\mathrm{CO}_{2}$ de cette génération de bioéthanol montrent qu'il ne serait pas la panacée pour résoudre le problème du réchauffement planétaire [4, 5]. Enfin, la production de ce carburant n'exploite qu'une partie de la plante cultivée et, de ce fait, constitue une utilisation sous-optimale des ressources en eau et des terres arables.

A contrario, la production d'éthanol à partir de la biomasse lignocellulosique, une ressource abondante et renouvelable, constitue une piste intéressante et un enjeu majeur pour le futur développement de biocarburants comme alternatives crédibles pour les énergies d'origine fossile.

\section{Le concept de l'éthanol cellulosique}

La fermentation du glucose en éthanol est une pratique humaine millénaire qui, au fur et à mesure du temps, s'est transformée en technologie industrielle. En raison certainement de la grande maturité de cette industrie, le concept du raffinage de la biomasse lignocellulosique est, la plupart du temps, réduit à un concept de transformation de la cellulose en éthanol. Par conséquent, les recherches qui ont été conduites depuis une trentaine d'années ont porté surtout sur les défis liés à la mise en condition de la cellulose, à son hydrolyse et à la fermentation des sirops de glucose produits par ces opérations. Aujourd'hui, le schéma opérationnel considéré comme étant le plus prometteur fonctionne en trois étapes : le prétraitement de la matière brute, I'hydrolyse enzymatique de la cellulose et la fermentation des hydrolysats.

\section{La structure de la biomasse lignocellulosique}

La biomasse lignocellulosique est une matière composite dont les principaux constituants sont la cellulose, les hémicelluloses et les lignines. À l'intérieur de la biomasse lignocellulosique, ces trois macromolécules s'entremêlent et forment une structure tridimensionnelle complexe et très résistante, maintenue par des liaisons hydrogène et des liaisons covalentes, qui résiste aux attaques de phytopathogènes et qui confère de la rigidité aux plantes. La proportion et la nature de chacune des macromolécules sont fonction de l'origine botanique 
Tableau 1. Composition en glucides de matières lignocellulosiques d'origine botanique différente.

\begin{tabular}{|lccc|}
\hline & \multicolumn{3}{c|}{ \% Matière sèche } \\
Matière & $\boldsymbol{\beta}$-Glucane & Hémicellulose & Lignine \\
\hline Maïs (tige et feuille) & 38 & $25^{\text {a }}$ & 19 \\
Paille de blé & 33 & $23^{\text {a }}$ & 17 \\
Paille de riz & 39 & $15^{\text {a }}$ & 10 \\
Paille de l'orge & 40 & $19^{\text {a }}$ & 14 \\
Pin de Monterrey & 42 & $21^{\text {b }}$ & 26 \\
Peuplier hybride & 41 & $22^{\text {c }}$ & 24 \\
Sorgho (fourrage) & 34 & $17^{\text {a }}$ & 16 \\
Bagasse & 39 & $23^{\text {a }}$ & 23 \\
\hline
\end{tabular}

${ }^{\text {a }}$ Arabinoxylane.

${ }^{\mathrm{b}}$ Majoritairement galactomannane.

c Arabino-4-0-methyl glucuronoxylane.

de la matière et des différences importantes sont possibles (tableau 1).

\section{La mise en condition ou le prétraitement de la cellulose}

Aujourd'hui, le prétraitement de la matière lignocellulosique est l'opération unitaire la plus coûteuse dans la transformation de la biomasse en éthanol [6]. Néanmoins, elle est absolument indispensable, car les cellulases dont nous disposons sont très peu actives sur la biomasse à l'état brut [7]. La fonction primaire du prétraitement est donc de diminuer la complexité de la biomasse et d'augmenter l'efficacité des cellulases [8]. Pour ce faire, un bon prétraitement doit exposer la cellulose en éliminant les hémicelluloses qui lui sont associées; diminuer la cristallinité de la cellulose et créer des zones amorphes ; éliminer ou modifier les lignines qui entravent l'action des cellulases employées dans l'étape suivante. En même temps, pour qu'un prétraitement soit utile et efficace, il est important d'établir des conditions limitant la formation de produits de dégradation qui peuvent inhiber l'étape de fermentation [9-11] et de mettre en œuvre des technologies qui ne nécessitent pas une étape préalable de broyage. Par ailleurs, le catalyseur utilisé pour le prétraitement doit être de faible coût, ou bien recyclable, et le résultat net du procédé doit être l'augmentation de la valeur des différents composants de la biomasse. Dans la réalité, ces critères sont parfois antagonistes et les technologies actuelles constituent des compromis. Les prétraitements actuellement en développement utilisent comme catalyseurs des produits chimiques. Ainsi, les méthodes les plus fréquentes mettent en œuvre une catalyse acide, mais la catalyse alcaline et la catalyse OrganoSolv sont aussi assez répandues.

La catalyse acide, étudiée depuis de nombreuses années, utilise le plus souvent de l'acide sulfurique ou de l'acide chlorhydrique à faible concentration ( 0,5 à $1,5 \%)$ et des températures supérieures à $140{ }^{\circ} \mathrm{C}[12]$. Dans ces condi- tions, les hémicelluloses, plus labiles que la cellulose, sont dépolymérisées et des résidus pentoses, sous la forme d'oligomères ou de monomères, sont obtenus. Selon la sévérité des conditions employées, une partie des résidus pentoses peut subir une réaction de déshydratation qui conduit à la formation de furfural. La manière exacte de mettre en œuvre ce prétraitement acide est très variable, mais il a été souvent combiné avec une explosion à la vapeur. Cette technologie, développée au Canada par EA De Long, s'effectue dans un réacteur sous pression $\left(>2 \times 10^{6} \mathrm{~Pa}\right)$ à des températures allant de 160 à $240^{\circ} \mathrm{C}[13,14]$. La biomasse est incubée dans ces conditions pendant plusieurs dizaines de secondes (jusqu'à 1 min 30 s), puis la réaction est arrêtée par une décompression rapide jusqu'à la pression atmosphérique, ce qui conduit à la désintégration de la matière. La combinaison de I'explosion à la vapeur et de la catalyse acide est des plus efficaces, car elle permet d'obtenir $>70 \%$ des résidus xylose sous la forme de sucres monomères. Néanmoins, comme les autres méthodes à catalyse acide, elle nécessite I'utilisation d'équipements coûteux afin $\mathrm{d}^{\prime}$ atténuer les problèmes liés à la corrosivité $\mathrm{du}$ catalyseur.

Les méthodes alcalines n'ont pas le même mode d'action que la catalyse acide. L'emploi de bases fortes conduit à une très bonne solubilisation des hémicelluloses et à la modification des lignines, car il permet de saponifier les liaisons esters qui contribuent à la structuration du réseau pariétal. Cependant, ces catalyseurs sont généralement plus chers que les acides et, par conséquent, une stratégie de recyclage s'impose. Par ailleurs, à la différence des acides, les alcalins ne catalysent pas I'hydrolyse des liaisons glycosidiques et ne dégradent pas les résidus pentoses en furfural. Par conséquent, les hémicelluloses sont obtenues sous forme de polymères solubles ou d'oligomères qui nécessitent d'autres modifications afin de produire un sirop de pentoses fermentescibles. En résumé, même si la catalyse alcaline présente des avantages par rapport à la catalyse acide, les bilans financiers des différentes méthodes sont au final assez semblables et pèsent toujours trop dans le coût final de l'éthanol [15].

\section{L'hydrolyse de la cellulose par voie enzymatique}

Dans cette étape du procédé, la cellulose est transformée en glucose selon la formule :

$\left[\mathrm{C}_{6} \mathrm{H}_{12} \mathrm{O}_{5}\right]_{n}+n \mathrm{H}_{2} \mathrm{O} \rightarrow n \mathrm{C}_{6} \mathrm{H}_{12} \mathrm{O}_{5}$.

Cette hydrolyse peut être catalysée par voie acide, mais aujourd'hui, à l'instar de la transformation industrielle de l'amidon en glucose, les enzymes constituent les catalyseurs de choix. Actuellement, le développement de cette technologie se focalise sur deux stratégies différentes : I'hydrolyse et la fermentation séparée (SHF en anglais) ou l'hydrolyse et la fermentation simultanée (ou SSF en anglais) (figure 1). La première est conceptuellement plus simple, car elle concerne la production par voie enzymatique d'un sirop de glucose qui est ensuite transformé en éthanol par voie fermentaire, alors que la deuxième stratégie favorise l'intégration de deux procédés, puisque I'hydrolyse enzymatique est réalisée dans le bioréacteur en présence du micro-organisme fermentaire. Le procédé SSF est théoriquement meilleur, car il permet de réduire les coûts d'opération et de palier les problèmes liés à l'inhibition des enzymes par leurs produits [16]. Cependant, il nécessite une bonne compatibilité des biocatalyseurs (enzymes et micro-organisme fermentaire), ce qui n'est pas encore le cas aujourd'hui.

Quant aux enzymes mises en œuvre, la norme industrielle aujourd'hui repose sur I'utilisation des enzymes secrétées par le champignon cellulolytique, Trichoderma reesei. Ce champignon découvert par l'armée américaine pendant la seconde guerre mondiale, produit un vaste arsenal d'enzymes (connues sous le terme générique de cellulases) qui dégradent la biomasse, et en particulier la cellulose. Trois types d'activités enzymatiques sont principalement responsables de son action (figure 2). II s'agit des endoglucanases, des cellobiohydrolases et des $\beta$-glucosidases [17]. Les premières hydrolysent les liaisons $\beta-1,4$ qui relient des unités glucose à l'intérieur des chaînes de cellulose, et génèrent des extrémités réductrices qui servent comme points d'attaque pour les cellobiohydrolases. Celles-ci produisent du cellobiose (contenant deux molécules de glucose) qui sert ensuite de substrat pour les $\beta$-glucosidases qui, à leur tour, génèrent du glucose.

Aujourd'hui, le problème majeur de cette étape de saccharification enzymatique de la cellulose concerne le coût élevé des enzymes. Ceci est dû à la relative inefficacité des cellulases. Par comparaison avec les amylases qui sont employées pour fabriquer de l'éthanol 1 ère 


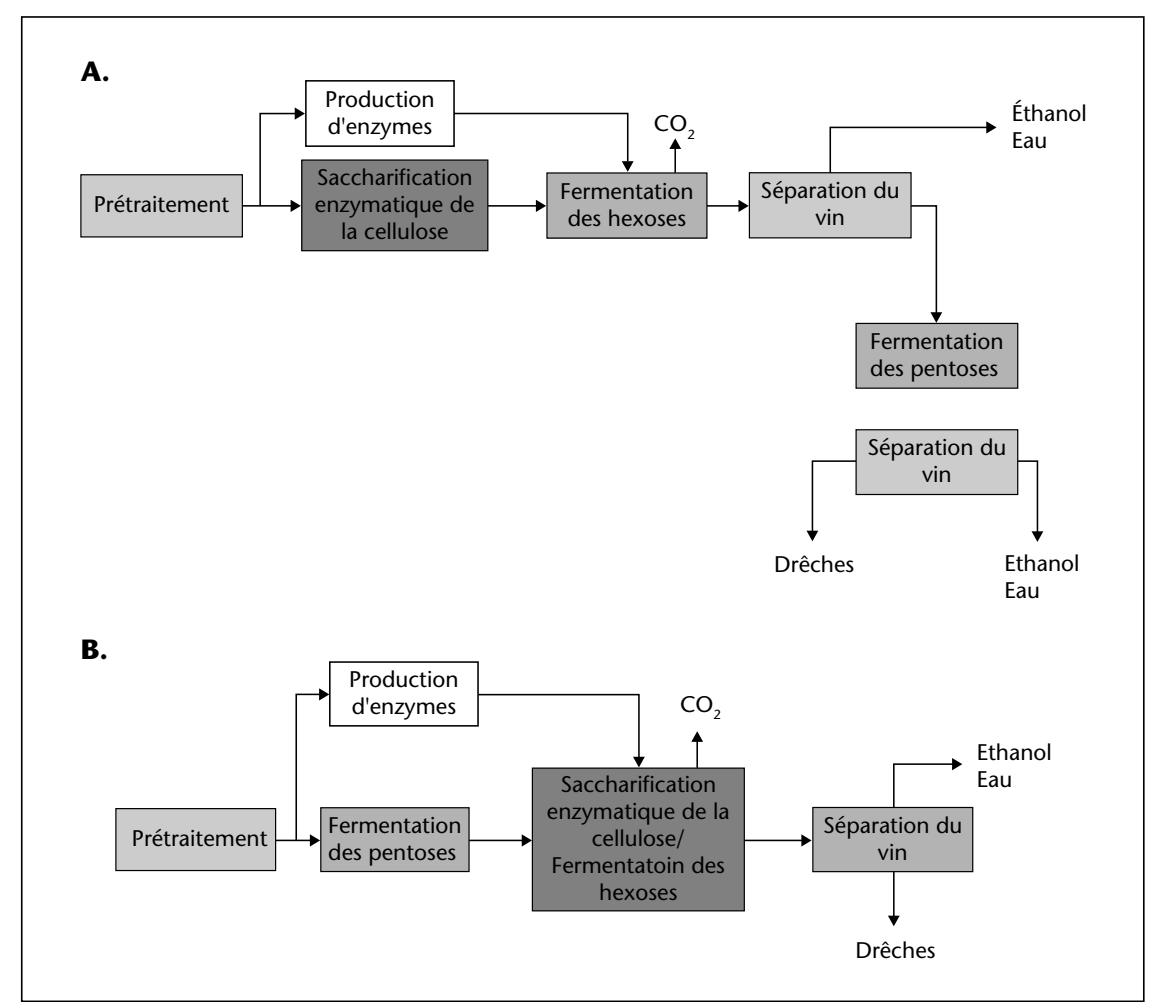

Figure 1. Deux niveaux d'intégration des opérations unitaires. A) L'hydrolyse et la fermentation séparée (SHF en anglais) ou B) I'hydrolyse et la fermentation simultanée. Dans les deux cas, il est envisageable d'utiliser les pentoses comme substrat pour la production d'enzymes.

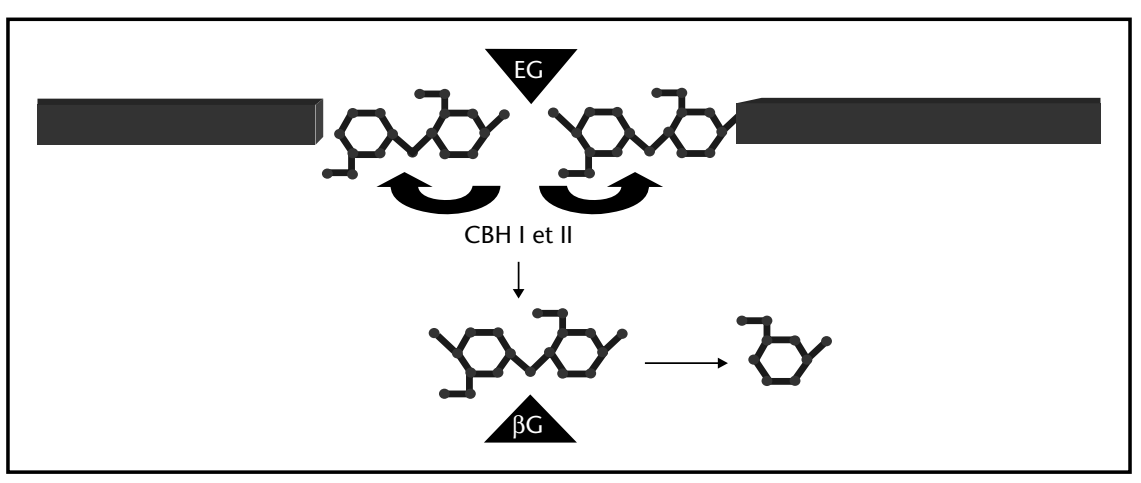

Figure 2. Les trois types d'activités enzymatiques requises pour la saccahrifcation de la cellulose. EG ; $\beta$ - $(1,4)$ endoglucanase ; $\beta-G ; \beta-(1,4)$-glucosidase et $C B H$; cellobiohydrolase.

génération à partir d'amidon, il est estimé qu'il faut 40 à 100 fois plus de cellulases pour produire la même quantité d'éthanol. Par conséquent, depuis de nombreuses années d'importants efforts ont été déployés pour améliorer les cellulases [17].

Ainsi, pour diminuer le coût des cellulases, différentes stratégies ont déjà été adoptées, dont certaines visent à améliorer l'efficacité intrinsèque de ces enzymes. À forte concentration en glucides, les cellulases de $T$. reesei sont inhibées par leurs produits, le cellobiose et le glucose. Par conséquent, il est utile soit de modifier ces enzymes est déterminante pour obtenir des rendements élevés. Or, dans les mélanges cellulolytiques provenant de souches naturelles, cette synergie n'est pas nécessairement optimale. Par conséquent, un effort considérable de recherche a consisté en l'amélioration ou le renforcement de certaines activités. Soit il s'agit d'augmenter l'expression et donc la quantité d'un des composants enzymatiques, soit il s'agit d'ajouter une nouvelle enzyme provenant d'une autre source microbienne. À titre d'exemple, dans une étude réalisée par Boisset et al., il a été montré que la mise en œuvre simultanée des cellobiohydrolases Cel7A de T. reesei et Cel 6A de Humicola insolens permet une synergie renforcée [22]. De même, dans une autre étude, des résultats probants ont été obtenus par le renforcement de l'activité $\beta$-glucosidase, qui est essentielle pour éviter une accumulation du cellobiose et une inhibition des cellobiohydrolases.

Hormis les cellulases, il a été clairement démontré que d'autres enzymes sont aussi importantes pour la transformation efficace de la biomasse en sirops de sucres fermentescibles. Parmi celles-ci l'on peut citer les xylanases, les xylosidases et les arabinofuranosidases qui agissent sur les arabinoxylanes (les hémicelluloses majeurs chez les graminées et le bois de feuillu) $[23,24]$. Ces enzymes sont particulièrement utiles lorsqu'il s'agit de l'hydrolyse d'une cellulose préparée par prétraitement alcalin, car comme nous l'avons déjà précisé, ce prétraitement ne permet pas de dépolymériser totalement les hémicelluloses [25]. Par ailleurs, ces enzymes sont particulièrement cruciales lors du traitement des biomasses riches en hémicelluloses telles que certains coproduits agricoles comme le son de blé et les rafles de mais [26].

Depuis 2001, les spécialistes industriels des enzymes tels que Novozymes ou DaniscoGenencor ont été contractés par le gouvernement américain pour développer et produire des cellulases à prix compétitif ${ }^{1}$. Aujourd'hui, I'objectif est d'atteindre un coût cible de l'ordre de $0,05 €$ /litre d'éthanol. II est estimé qu'à court terme cet objectif est atteignable, surtout si la production des enzymes est réalisée à proximité des unités de production d'éthanol.

enzymes par mutagenèse pour lever cette tation, soit d'éliminer le glucose de façon continue, par exemple en utilisant la technique SSF. Via la technique de mutagenèse in vivo de la souche entière ou celle de la modification in vitro des enzymes, des avancées dans ce domaine, et vis-à-vis d'autres aspects, ont été réalisées [18-21]. Une autre stratégie qui vise à améliorer la performance des cellulases consiste à renforcer leur action synergique. Comme nous l'avons vu, l'hydrolyse de la cellulose requiert une cascade enzymatique où une bonne coopération entre les différentes

\section{La fermentation des sirops complexes}

La fermentation alcoolique est une technologie millénaire et constitue l'application biotechnologique la plus robuste. Néanmoins, depuis presque quarante ans, la fermentation alcoolique des sirops de sucres obtenus à partir de la

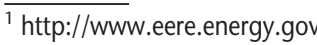


biomasse fait l'objet de recherches intensives et continues encore aujourd'hui, de poser des problèmes. L'origine de ce constat paradoxal provient de la complexité de la biomasse. Comme il a été dit précédemment, à la différence du saccharose extrait de la canne à sucre ou de la betterave sucrière, la biomasse lignocellulosique est une matière première complexe et variable, composée de polysaccharides et de lignines. En plus du glucose, qui est facilement fermenté par la levure Saccharomyces cerevisiae (la norme industrielle pour la fermentation alcoolique), les autres hexoses tels que le galactose et le mannose sont moins bien fermentés et, surtout, les pentoses (xylose et arabinose) ne sont même pas assimilés par S. cerevisiae. Par ailleurs, selon la méthode de prétraitement employée pour extraire la cellulose, de nombreuses molécules de faible poids moléculaire peuvent être présentes dans les sirops fermentescibles. II s'agit entre autres de l'acide acétique, du furfural et du 5-Hydroxy(Méthyl-2)-furfural et de divers composés phénoliques qui sont tous des inhibiteurs de la fermentation [10]. La solution la plus simple pour palier l'ensemble de ces problèmes consiste en la purification/détoxification des sirops de glucose. Cependant, ces opérations de purification sont coûteuses et augmentent de manière sensible (> $20 \%$ ) le coût de production de l'éthanol, qui reste tout de même un produit de faible valeur commerciale [27]. En admettant qu'il est préférable d'éviter ces étapes de purification, plusieurs développements s'imposent pour lever les différents verrous déjà évoqués. $D^{\prime}$ abord, il est indispensable d'intégrer les pentoses dans les processus de transformation soit pour produire de l'éthanol, soit pour la production d'autres produits commerciaux. Ensuite, l'identification de nouvelles souches fermentaires qui présentent une tolérance élevée à la présence d'inhibiteurs s'avère incontournable.

La recherche de souches capables de transformer les pentoses en éthanol a débuté dans les années 70. Depuis, les travaux réalisés ont surtout porté, d'une part, sur l'élaboration de souches de $S$. cerevisiae génétiquement modifiées ayant l'aptitude d'assimiler le xylose et, d'autre part, sur l'identification et le développement de nouvelles souches microbiennes pour la fermentation alcoolique. En ce qui concerne I'ingénierie de $S$. cerevisiae, une découverte des années 70 a montré que cette levure est capable de fermenter le xylulose en éthanol, ouvrant une voie vers I'amélioration de cette souche. En effet, le xylose pouvant être transformé en xylulose par l'action d'une enzyme, la xylose isomérase (XI), l'introduction $d^{\prime}$ 'une telle activité enzymatique chez $S$. cerevisiae permettrait à cette souche d'assimiler et de fermenter le xylose. Malheureusement, il n'en est rien, car il s'est avéré difficile d'exprimer un gène codant pour une XI chez $S$. cerevisiae. De surcroît, dans les cas où cet exploit a tout de même été réalisé, il est devenu clair que les autres activités enzymatiques impliquées dans la voie des pentoses phosphates et nécessaires à cette conversion sont trop peu exprimées chez S. cerevisiae. Pire encore, lorsque l'expression de toutes les activités enzymatiques de cette voie a été augmentée, la fermentation de xylose en éthanol est restée faible [28], car en réalité il est nécessaire d'employer une approche holistique, capable de prendre en compte toute la complexité du système.

Face aux échecs d'amélioration de la levure S. cerevisiae, de nombreux chercheurs se sont tournés vers d'autres micro-organismes tels Zymomonas mobilis, Escherichia coli et Klebsiella oxytoca $[29,30]$. La bactérie $Z$. mobilis est singulière car elle est capable de produire de l'éthanol uniquement à partir du glucose, du fructose et du saccharose, avec des performances qui dépassent parfois celles de S. cerevisiae. Cependant, comme cette levure, Z. mobilis ne fermente ni le xylose ni l'arabinose et présente une gamme de substrats très étroite, et à nouveau, de nombreuses recherches ont porté sur la modification génétique de cette bactérie. À l'inverse, $E$. coli et $K$. oxytoca utilisent une large gamme de glucides comme substrats, mais présentent des tolérances à l'éthanol faible. Dernièrement, de nombreuses recherches sur le développement de différentes bactéries comme souches productrices d'éthanol ont été entreprises, mais les cultures bactériennes demeurent moins robustes que celles de S. cerevisiae, qui reste la norme industrielle.

Depuis quelques années, les chercheurs ont réalisé des progrès considérables pour améliorer S. cerevisiae, grâce notamment à une progression des connaissances fondamentales, au développement des approches génomique, protéomique et transcriptomique et à l'introduction de nouvelles techniques pour l'ingénierie métabolique [28, 31-38]. Aujourd'hui de nombreuses souches de laboratoires très performantes existent, mais ces levures ne sont pas encore adaptées à des conditions industrielles, car elles ne tolèrent pas la présence d'inhibiteurs présents dans les hydrolysats de lignocelluloses [39]. Par ailleurs, les conditions de fermentation à l'échelle industrielle sont parfois très différentes de celles utilisées en laboratoire. En particulier, le manque ou l'absence totale de stérilité en milieu industriel conduit à l'apparition de contaminants (exemple : souches sauvages de S. cerevisiae, Dekkera bruxellensis, Candida sp.) qui influencent, de manière significative, le profil dynamique de la fermentation et, in fine, la productivité du processus $[40,41]$. Par conséquent, les futurs développements de levures industrielles devront aussi prendre en compte cette dimension complexe de la réalité industrielle.

\section{Vers le développement de procédés moins coûteux et plus propres}

En biologie, le XXe siècle a été une période de grand progrès. La biochimie moderne est née, la structure de I'ADN a été découverte et le séquençage et l'amplification in vitro de I'ADN ont été mis au point. Ces avancées, et d'autres encore, contribuent aujourd'hui à la forte progression des biotechnologies dans tous les secteurs et permettent de postuler que d'ici quelques années la transformation de la biomasse en éthanol s'effectuera par un procédé biotechnologique intégré où la consommation en produits chimiques, en énergie et en eau sera réduite à un strict minimum. Les premiers signes de cet avenir sont nombreux, et les progrès réalisés dans la transformation industrielle de l'amidon en sirop de dextrose sont à ce titre pertinents.

Ainsi, la commercialisation à partir des années 1960 d'amylases thermostables capables de liquéfier l'amidon à $90^{\circ} \mathrm{C}$ a déclenché le recul de l'hydrolyse acide comme pratique courante dans I'industrie de I'amidon. Des recherches successives ont permis d'améliorer ces enzymes au niveau de leurs propriétés physicochimiques, de telle sorte qu'aujourd'hui la plupart des sirops de dextrose produits dans le monde sont fabriqués par un procédé enzymatique qui nécessite la gélification initiale des grains d'amidon à haute température. Récemment, un nouveau concept pour la transformation de l'amidon a encore été proposé, reposant sur I'hydrolyse enzymatique directe des grains d'amidon à basse température $[42,43]$. Cette nouvelle technologie, moins consommatrice en énergie et adaptée à la fabrication d'éthanol à partir de céréales, provient de recherches focalisées, d'une part, sur l'identification dans la biodiversité de nouveaux catalyseurs et, d'autre part, sur l'ingénierie moléculaire d'enzymes amylolytiques qui présentent une aptitude naturelle pour l'hydrolyse directe des grains d'amidon. La mise au point de cette méthode ouvre désormais la voie vers un procédé intégré où la liquéfaction, la saccharification et la fermentation peuvent être réalisées simultanément.

À l'instar de ce qui vient d'être décrit, il est certain que les nouvelles méthodes biotechnologiques qui permettent de prospecter la biodiversité (l'approche métagénomique) [44, 45] et les diverses techniques d'ingénierie moléculaire qui favorisent l'adaptation des enzymes aux contraintes industrielles (l'évolution dirigée et l'ingénierie rationnelle) [46] joueront 
des rôles majeurs pour la production de l'éthanol de $2^{\mathrm{e}}$ génération.

\section{Conclusion}

Aujourd'hui, le blé (grains) se négocie en France (Rouen) à $230 € /$ tonne. Cette hausse récente du prix du blé s'inscrit dans une tendance mondiale à l'augmentation générale des denrées alimentaires de base, notamment les céréales. Les raisons de cette flambée sont multiples et ne sont pas uniquement liées à la production d'agrocarburant. Néanmoins, le résultat conduit surtout à un accroissement de la pauvreté dans de nombreux pays où l'équilibre social est déjà fragile. Par conséquent, dans ce contexte international tendu, la recherche visant la mise au point de nouvelles technologies pour la production de l'éthanolcarburant à partir de matières premières non alimentaires (biomasse lignocellulosique) constitue plus que jamais un enjeu majeur pour la société et un objectif important pour la communauté scientifique.

Au-delà du simple objectif de production d'agrocarburants à partir de matière lignocellulosique, la bonne gestion des ressources en biomasse est aussi un défi pour demain. Pour y faire face, un changement de paradigme sera sans doute nécessaire, car jusqu'ici la recherche et le développement de technologies pour la transformation de la biomasse sont focalisés sur l'extraction du glucose pour la fabrication de l'éthanol. À l'avenir, il faudra plutôt considérer l'ensemble de la matière végétale comme une ressource précieuse, nécessitant une déstructuration maîtrisée, afin d'extraire chacun des composants qui seront ensuite valorisés individuellement pour décliner une large gamme de produits [47]. Pour atteindre cet objectif ambitieux et réduire la consommation en énergie et en catalyseurs chimiques, il faudra sans aucun doute puiser dans la biodiversité pour trouver de nouvelles enzymes et appliquer la pleine puissance des biotechnologies pour adapter ces biocatalyseurs aux besoins de l'industrie.

\section{RÉFÉRENCES}

1. VILLE J, MESTRAZAT W. Sur I'hydrolyse fluorhydrique de la cellulose. Comptes rendus de I'Académie des Sciences $1910 ; 150$ : 783-4.

2. HERRERA S. Bonkers about biofuels. Nat Biotechnol 2006 ; 24 : 755-60.

3. ANONYMOUS. Fuel ethanol output seen above SNPAA's estimate. FO Licht's world ethanol and biofuels report $2008 ; 6: 240$.

4. VON BLOTTNITZ H, CURRAN MA. A review of assessments conducted on bio-ethanol as a transportation fuel from a net energy, greenhouse gas, and environmental life cycle perspective. / Clean Prod 2007; 15 : 607.
5. WANG M, WU M, HONG H. Life-cycle energy and greenhouse gas emission impacts of different corn ethanol plant types. Environ Res Let $2007 ; 2: 1-13$.

6. WYMAN CE. What is (and is not) vital to advancing cellulosic ethanol. Tren Biotechnol 2007 ; $25: 153-7$

7. WRIGHTJD, WYMANCE, GROHMANN K. Simultaneous saccharification and fermentation of lignocellulose - process evaluation. App BiochemBiotechnol $1988 ; 18$ : 75-90.

8. MOSIER N, WYMAN C, DALE B, ET AL. Features of promising technologies for pretreatment of lignocellulosic biomass. Bioresour Technol $2005 ; 96: 673-86$

9. KABEL MA, BOS G, ZEEVALKING J, VORAGENAG], SCHOLS HA. Effect of pretreatment severity on xylan solubility and enzymatic breakdown of the remaining cellulose from wheat straw. Bio Technol 2007 ; $98: 2034$

10. PALMQVIST E, HAHN-HAGERDAL B. Fermentation of lignocellulosic hydrolysates. II : inhibitors and mechanisms of inhibition. Bio Technol $2000 ; 74: 25-33$.

11. LARSSON S, PALMQVIST E, HAHN-HAGERDAL B, ET AL. The generation of fermentation inhibitors during dilute acid hydrolysis of softwood. Enz Microb Technol 1999 ; 24 : 151-9.

12. LEE YY, PRASHANT I, TORGET RW. Dilute-acid hydrolysis of lignocellulosic biomass. In : Scheper T, ed. Advances in Biochemical Engineering/ Biotechnology and Bioengineering, Vol. 65. Berlin Heidelberg : Springer-Verlag, 1999 : 94-115.

13. DELONG EA, RITCHIE GS. inventors. (1990). US Patent 4966650

14. GALBE M, ZACCHI G. Pretreatment of lignocellulosic materials for efficient bioethanol production. Biofuels 2007 ; $108: 41-65$.

15. EGGEMAN T, ELANDER RT. Process and economic analysis of pretreatment technologies. Bio Technol $2005 ; 96: 2019-25$.

16. CARDONA CA, SANCHEZ OJ. Fuel ethanol: Process design trends and integration opportunities. Bio Technol 2007 ; 98 : 2415-57.

17. MERINO ST, CHERRY J. Progress and challenges in enzyme development for Biomass utilization. Biofuels 2007 ; 108 : 95-120.

18. GOEDEGEBUUR F, DANKMEYER L, GUALFETTI P, ET AL. Improving the thermal stability of cellobiohydrolases I (Cel7A) from $T$. reesei by site directed evolution. J Biotechnol $2005 ; 118$ : S130.

19. SANDGREN M, STAHLBERG ], MITCHINSON C. Structural and biochemical studies of $\mathrm{GH}$ family 12 cellulases : improved thermal stability, and ligand complexes. Prog Biophys Mol Biol 2005 ; 89 : 246-91.

20. MITCHINSON C. Improved cellobiohydrolases for biomass conversion. Prot Sc $2004 ; 13: 212$.
21. GADGIL NJ, DAGINAWALA HF, CHAKRABARTI T, KHANNA P. Enhanced cellulase production by a mutant Of Trichoderma reesei. Enz Microb Technol 1995 ; 17 : 942-6.

22. BOISSET C, FRASCHINI C, SCHULEIN M, HENRISSAT B, CHANZY H. Imaging the enzymatic digestion of bacterial cellulose ribbons reveals the endo character of the cellobiohydrolase Cel6A from Humicola insolens and its mode of synergy with cellobiohydrolase Cel7A. Appl Environ Microbiol 2000 ; 66 : 1444-52.

23. KULKARNI N, SHENDYE A, RAO M. Molecular and biotechnological aspects of xylanases. FEMS Microbiol Rev 1999 ; 23 : 411-56.

24. SAHA BC. alpha-L-arabinofuranosidases: biochemistry, molecular biology and application in biotechnology. Biotechnol Adv 2000; 18 : 403-23.

25. WYMAN CE, DALE BE, ELANDER RT, HOLTZAPPLE M, LADISCH MR, LEE YY. Comparative sugar recovery data from laboratory scale application of leading pretreatment technologies to corn stover. Bioresour Technol $2005 ; 96$ : 202632.

26. SORENSEN HR, PEDERSEN S, JORGENSEN CT, MEYER AS. Enzymatic hydrolysis of wheat arabinoxylan by a recombinant "minimal" enzyme cocktail containing beta-xylosidase and novel endo-1,4-beta-xylanase and alpha-(L)-arabinofuranosidase activities. Biotechnol Prog 2007; $23: 100-7$.

27. VON SIVERS M, ZACCHI G, OLSSON L, HAHNHAECERDAL B. Cost analysis of ethanol production from willow using recombinant Escherichia coli. Biotechnol Prog $1994 ; 10$ : 555-60.

28. HAHN-HAGERDAL B, KARHUMAA K, JEPPSSON M, GORWA-GRAUSLUND MF. Metabolic engineering for pentose utilization in Saccharomyces cerevisiae. Biofuels 2007 ; 108 : 147-77.

29. DIEN BS, COTTA MA, JEFFRIES TW. Bacteria engineered for fuel ethanol production : current status. Appl Microbiol Biotechnol $2003 ; 63$ : 258-66.

30. ROGERS PL, JEON YJ, LEE KJ, LAWFORD HG. Zymomonas mobilis for fuel ethanol and higher value products. Biofuels $2007 ; 108: 263-88$.

31. VAN MARIS AJA, WINKLER AA, KUYPER M, DE LAAT W, VAN DIJKEN JP, PRONK JT. Development of efficient xylose fermentation in Saccharomyces cerevisiae: Xylose Isomerase as a key component. Biofuels 2007 ; 108 : 179-204.

32. VAN MARIS AIA, ET AL. Alcoholic fermentation of carbon sources in biomass hydrolysates by Saccharomyces cerevisiae : current status. Antonie Van Leeuwenhoek. Int / Gen Mol Microbiol $2006 ; 90: 391-418$.

33. KUYPER M, HARTOG MMP, TOIRKENS M], ETAL. Metabolic engineering of a xyloseisomerase-expressing Saccharomyces cerevisiae strain for rapid anaerobic xylose fermentation. FEMS Yeast Res 2005 ; 5 : 399-409. 
34. KUYPER M, TOIRKENS MI, DIDERICH JA, WINKLER AA, VAN DIJKEN JP, PRONK JT. Evolutionary engineering of mixed-sugar utilization by a xylose-fermenting Saccharomyces cerevisiae strain. FEMS Yeast Res $2005 ; 5$ : 925-34.

35. KUYPER M, WINKLER AA, VAN DIJKEN JP, PRONK JT. Minimal metabolic engineering of Saccharomyces cerevisiae for efficient anaerobic xylose fermentation : a proof of principle. FEMS Yeast Res $2004 ; 4$ : 655-64.

36. KUYPER M, ET AL. High-level functional expression of a fungal xylose isomerase : the key to efficient ethanolic fermentation of xylose by Saccharomyces cerevisiae?. FEMS Yeast Res $2003 ; 4: 69-78$.

37. WISSELINK HW, TOIRKENS M], BERRIEL MDF, ET AL. Engineering of Saccharomyces cerevisiae for efficient anaerobic alcoholic fermentation of L-arabinose. Appl Environ Microbiol 2007 ; 73 : 4881-91.
38. PRONK J, KUYPERM, TOIRKENSM, WINKLER R, VAN DIJKEN H, DE LAAT W. Engineering Saccharomyces cerevisiae for xylose utilization. J Biotechnol $2005 ; 118$ : S86-S87.

39. HAHN-HÄGERDAL B, KARHUMAA K, FONSECAC, SPENCER-MARTINSI, GORWAGRAUSLUND M. Towards industrial pentosefermenting yeast strains. Appl Microbiol Biotechnol $2007 ; 74$ : 937.

40. BASÍLIO A, DE ARAÚJO P, DE MORAIS J, DA SILVA FILHOE, DE MORAIS M, SIMÕES D. Detection and identification of wild yeast contaminants of the industrial fuel ethanol Fermentation Process. Curr Microbiol 2008; 56 : 322.

41. DE SOUZA LIBERAL AT, BASILIO ACM, DO MONTE RESENDE A, ET AL. Identification of Dekkera bruxellensis as a major contaminant yeast in continuous fuel ethanol fermentation. I Appl Microbiol 2007 ; 102 : 538-47.

42. WILLIAMS J. Break it down now. Ethanol Producer Magazine, January 2006.
43. ROBERTSON GH, WONG DWS, LEE CC, WAGSCHAL K, SMITH MR, ORTS W]. Native or raw starch digestion: A key step in energy efficient biorefining of grain. J Agr Food Chem $2006 ; 54: 353-65$.

44. BULL AT, WARD AC, GOODFELLOW M. Search and discovery strategies for biotechnology: the paradigm shift. Microbiol Mol Biol Rev 2000 ; $64: 573-606$

45. SCHMEISSER C, STEELE H, STREIT WR. Metagenomics, biotechnology with non-culturable microbes. Appl Microbiol Biotechnol 2007 ; 75 : 955-62.

46. BLOOM JD, MEYER MM, MEINHOLD $P$, OTEY CR, MACMILLAN D, ARNOLD FH. Evolving strategies for enzyme engineering. Curr Opin Str Biol $2005 ; 15: 447$.

47. ZHANG YH. Reviving the carbohydrate economy via multi-product lignocellulose biorefineries. J Industr Microbiol Biotechnol 2008 ; 35 : 367. 